\title{
ACTonHEALTH study protocol: promoting psychological flexibility with activity tracker and mHealth tools to foster healthful lifestyle for obesity and other chronic health conditions
}

Roberto Cattivelli ${ }^{1,2^{*}}$ D, Gianluca Castelnuovo ${ }^{1,2}$, Alessandro Musetti ${ }^{3}$, Giorgia Varallo ${ }^{1,2}$, Chiara A. M. Spatola ${ }^{1,2}$, Francesco Vailati Riboni ${ }^{2}$, Anna Guerrini Usubini ${ }^{1}$, Fabio Tosolin ${ }^{5}$, Gian Mauro Manzoni ${ }^{1,4}$, Paolo Capodaglio ${ }^{7}$, Alessandro Rossi ${ }^{1,6}$, Giada Pietrabissa ${ }^{1,2}$ and Enrico Molinari ${ }^{1,2}$

\begin{abstract}
Background: Obesity and the state of being overweight are increasing steadily and becoming a global epidemic. Recent research reports $64 \%$ of the adult population as overweight in Europe and the USA. The social and economic impacts are increasing, and most of the rehabilitation programs, while effective in the short term, do not produce long-lasting results. An explanatory model from a behavioral perspective can describe the phenomena with the lack of sources of reinforcement related to healthful habits in a daily life context.
\end{abstract}

Methods/design: A randomized clinical trial combining single-subject studies and a four-arm group design will be conducted to compare the effect of the current standard in obesity treatment to Acceptance and Commitment Therapy (ACT) and wearable technology at different times, before starting intervention, at the end, and at follow-up visits of 3, 6, and 12 months measuring changes over time of physical activity and psychological well-being.

Discussion: The goal of this project, combining ACT and wearable technology, is to develop an effective intervention, efficient and sustainable, which even after discharge can provide adequate contingencies of reinforcement in the natural environment, integrating systematic measurements, continuous feedback, and individualized, values-based objectives. The intervention is aimed to provide a contingent reinforcement for healthful behaviors instead of reinforcing only the achievement of a significant weight loss.

The aim of the project, combining Acceptance and Commitment Therapy and Wearable Technology, is to develop an effective, efficient and sustainable intervention able to provide a contingent reinforcement for healthy behaviors. The intervention is aimed to promote adequate healthy behaviors in the natural environment, integrating systematic measurements, continuous feedback and individualized values-based objectives, instead of reinforcing only the achievement of a significant weight loss.

Trial registration: ClinicalTrials.gov, NCT03351712. Registered on 24 November 2017.

Keywords: Acceptance and Commitment Therapy - ACT, Wearable, Activity trackers, Health promotion, Obesity, Chronic health condition, Behavior modification, Psychological flexibility, Randomized clinical trial, Multiple-baseline single-subject design

\footnotetext{
* Correspondence: r.cattivelli@auxologico.it

${ }^{1}$ Istituto Auxologico Italiano IRCCS, Psychology Research Laboratory,

Verbania, Italy

${ }^{2}$ Department of Psychology, Catholic University of Milan, Milan, Italy

Full list of author information is available at the end of the article
}

(c) The Author(s). 2018 Open Access This article is distributed under the terms of the Creative Commons Attribution 4.0 International License (http://creativecommons.org/licenses/by/4.0/), which permits unrestricted use, distribution, and reproduction in any medium, provided you give appropriate credit to the original author(s) and the source, provide a link to the Creative Commons license, and indicate if changes were made. The Creative Commons Public Domain Dedication waiver (http://creativecommons.org/publicdomain/zero/1.0/) applies to the data made available in this article, unless otherwise stated. 


\section{Background}

The condition of being overweight is an increasing problem worldwide and is becoming an epidemic both in Europe and the USA. According to recent estimates, more than $60 \%$ of adults are overweight, and obesity affects around $35 \%$ of the population [59]. In the USA, the healthcare system, in order to cope with obesity and related diseases, spends the sum of $\$ 100$ billion [24, 58, 84], and in Europe costs are currently similar [84] with a rising percentage [44]. Health risks related to being overweight include psychological difficulties, depression and stigma, physical problems and heart diseases, cancer, and respiratory diseases, not to mention musculoskeletal and metabolic problems [22, 23, 29, 41, 42, 55, 58, 75, 82].

Obesity is a chronic health condition [15], but it is also a major risk factor for various diseases, both chronic and acute [74]. This problem is currently rising, with prevalence data and epidemiological estimates that should be a matter of great concern [60]. Despite strong biological components and hereditary aspects, obesity is primarily linked to incorrect habits of everyday life, mainly eating and physical activity habits [16, 63]. Numerous studies $[29,45,59]$ have documented that regular physical activity and well-informed choices in food intake can prevent obesity and related conditions. The main challenge in addressing obesity and associated disorders is developing and providing sustainable comprehensive programs that include combinations of physical activity, dietary aspects, and psychological interventions [17-19]. Interventions developed in this way, including dietary education protocols, physical rehabilitation and exercise programs, and nutritional, endocrinal, psychological, surgical, and pharmacological treatments, are generally effective in the short term, despite many of them being characterized by high costs and long periods of hospitalization $[58,84,89]$. An analysis of the literature $[16,24,56]$ suggests the superiority of intensive multidisciplinary interventions: dietary programs and psychological and physical rehabilitation. One of the problems of such interventions is that they are more easily implementable during a hospitalization period, with high costs associated. However, despite good evidence of effectiveness, the long-term results are generally limited [27, 46]. In general, availability, costs, compliance, and long-term efficacy are important limitations to this variety of approaches [4, $11,15,20,25,64]$. Frequently, obese individuals will regain about $30 \%$ of the weight lost within the first year [28]. Often the inpatients return to pre-treatment weight after only 3 years [16]. Psychological interventions are generally assumed to be one of the core pillars in obesity treatment, supporting lifestyle change including dieting and physical activity. Among others, cognitive behavioral therapy (CBT) interventions are generally considered the gold standard for addressing "globesity" $[5,15]$. In particular, treatments aimed to promote a healthful lifestyle, with a strong focus on fostering physical activity, have proven efficacy $[43,54,91]$ and are generally considered the gold standard in the field [34, 39, 40, 72, 90]. CBT-based programs show generally good outcomes; nevertheless, according to recent research, the outcomes of these programs usually do not last in the long term [19, 40, 74, 80]. In order to maintain outcomes in a long-term perspective, recently acceptance and commitment therapy $(A C T)$ has been considered. Both as an add-on treatment and in a combined format, ACT could be beneficial specifically for improving long-term weight loss outcomes. Recent developments in mindfulness and acceptance-based interventions provide a potential avenue for treatment development [22].

\section{The present study}

Principal reasons for the long-term failure of obesity interventions are the lack of long-term sources of reinforcement for healthful habits acquired during the intensive treatment period [14, 31, 32]. The tradition of behavioral sciences has developed models of interpretation, explanation, and pragmatic operation supported by evidence of effectiveness and efficiency in the promotion of desired behaviors in various contexts, for example, smoke cessation $[10,81]$, pathological Internet use $[69,70]$, the ability to live with chronic diseases $[6,48,66]$, and the promotion of safety behaviors in working environments $[71,88]$. For this reason, behavior analysis in general, and in particular contextual behavioral science, can play a decisive role in the prevention of obesity and in the maintenance of the results achieved within short intensive protocols, usually held in hospitals. By modifying deeply rooted and long-term acquired habits, obese patients can obtain more psychological and physical health, reduce risk factors, and improve their overall health, wellness, and quality of life [53, 59] with benefits not only for the individual but also for his social environment. During rehabilitation patients affected by obesity are frequently in contact with contingencies extremely different from those occurring in their daily lives. During rehabilitation or hospitalization, contingencies are efficiently shaped to promote desired behaviors and decrease actions that are identified as dysfunctional, with both aversive and reinforcing contingencies. Later on, after the treatment, in the patient's natural environment initially behaviors are maintained, but since they were reinforced in an environment that provides adequate contingencies, they often undergo a process of extinction consequently with cessation of reinforcement [79]. At the same time, outside the hospital, dysfunctional behaviors, such as avoiding physical activity and eating tasty food, are reinforced in a systematic and 
contingent way. This tendency to extinction of functional and healthful habits and reinforcement of dysfunctional habits leads, in the long term, to gaining of the weight lost laboriously, which also leads to lower levels of well-being [77]. Although the existing programs already provide protocol phases to be implemented once patients are home, often the patients fail to adhere to nutrition programs and planned activities. On the contrary, they return frequently to previous habits and show a high dropout rate at follow-ups [76].

To overcome the limited long-term adherence after the hospitalization period, it is necessary to find a way to also give significant, contingent, and informative feedback after the rehabilitation phase. In this regard the use of activity trackers represents a clear, valid, and "smart" use of new technologies, linked to well-established behavioral science [78]. Regular physical activity is associated with numerous psychological and physical benefits. Physical activity maintenance over the long term is even more difficult to achieve, with a lead role assigned to motivation that is reported as an important factor for adherence to the initial decision to change the behavior as well as for actually maintaining the behavioral change. Smartphone technology provides an opportunity to deliver physical activity interventions remotely. Several studies have demonstrated the validity of the Fitbit activity monitor [1]. A "smart" physical activity application could track physical activity in real time using the built-in accelerometer, provide instant feedback on steps taken or calories burned, and deliver immediate reinforcement of physical activity. This application could also track progress toward physical activity goals over time and include other evidence-based features enabling goal setting and problem solving. Activity trackers could be used by healthcare workers and researchers to observe daily activities and make recommendations based on short- and long-term trends in behavior. These electronic devices could help consumers to monitor their health even outside the hospital. Therefore, newer activity trackers, such as the Fitbit, could be utilized in these types of intervention to facilitate self-monitoring and goal setting, which are essential for initiating and maintaining physical activity in the long term [12]. Fitbit provides a detailed but user-friendly report of physical activity data utilizing a color-coded scheme on the screen (for example, showing different colors of the activities for achieving daily goals). In addition, other features, such as smiley faces that appear when daily goals are achieved and badges awarded for specific accomplishments (for example, reaching a distance or step-count milestone), could be reinforcing and motivating for participants.

Perfectly incorporating the use of technology to provide meaningful feedback, treatments based on third-wave intervention, such as $\mathrm{ACT}$, or, more generally, acceptance and mindfulness-based approaches are promising to support long-lasting behavioral change in the field of healthful habits promotion [42, 46, 60, 61]. Mindfulness and accept ance-based interventions, often defined as third-generation behavioral approaches, have as their objective the modification of one's relationship to undesirable thoughts, feelings, or bodily sensations $([3,13,21,22,49,50,83])$. Recently, third-generation interventions have rapidly gained popularity. ACT, one of the new wave's most increasingly used interventions, is empirically supported for a wide variety of psychological and behavioral problems, including chronic pain, anxiety, depression, and smoking cessation $[3,13,21$, $22,49,50,83]$. ACT uses three cardinal processes (acceptance, mindfulness, and values) to promote psychological flexibility, definable as the ability to pursue values-based action even in the presence of unwanted thoughts, feelings, and bodily sensations. In the specific context of weight control, ACT aims to encourage healthful behavioral patterns in accordance with stated values, while providing mindfulness and acceptance skills to enhance commitment to values-based behavior $[7,61]$.

\section{Hypothesis, goals, and expected results}

The project aims to promote a long-term change in lifestyle as part of a multidisciplinary intervention, in close collaboration with physicians, the service of clinical nutrition, and osteoarticular rehabilitation.

The main goal of this study is test the efficacy of combining behavioral change technologies through contingent and meaningful feedback provided by wearable devices with an intervention based on ACT, a well-studied psychological treatment developed in a contextual behavioral framework. The combinations of this approach should be feasible and efficient, compared with the gold standard treatment, and have lower costs. Four experimental conditions are provided: (1) usual care during hospitalization with programmed follow-up checks, (2) contingent feedback on daily life activities through activity trackers or stand-alone wearable devices, (3) stand-alone ACT-based intervention, (4) a combination of the previous two conditions: ACT-based intervention and activity tracker with feedback.

The primary outcome, or dependent variable, of the study is level of physical activity as collected by wearable electronic devices, a largely used parameter in scientific investigations $[33,68]$. Other outcomes-for example, time of sleep-are collected by wearables, and others are provided by self-report through the web platform. The independent variable of the study is the type of treatment (ACT intervention and feedback provided by activity trackers). For the purpose of the study we collect data about daily steps and a workout $\log$ for each participant.

The intervention aims to help people in their everyday environment to more consistently keep the choices they have 
made and follow appropriate recommendations on diet and movement. The project is directed to participants in the multidisciplinary rehabilitation program at St. Joseph Hospital - Verbania, Istituto Auxologico Italiano, a private research institute that also works for the Italian national health system. The recruitment will take place progressively, and subjects will be randomized to the four experimental conditions set by the protocol. Data will be analyzed in different experimental single-subject over multiple-baseline designs $[2,8]$. The subjects are also grouped according to the experimental conditions to allow statistical analysis of experimental intergroup designs, consistent with the approach of a randomized controlled trial (RCT).

The CONSORT diagram of the study is shown in Fig. 1, and the SPIRIT schedule for this trial is given in Fig. 2, and a sample of wearable device is shown in Fig. 3. Table 1 illustrate the experimental conditions, and Additional files 1,2 and 3 are provided, consisting respectively in the complete SPIRIT checklist for the study, the Consort Full Checklist and the TIDieR checklist.

\section{Methods}

\section{Study design}

Recently the Oxford Centre for Evidence-Based Medicine (http://www.cebm.net/) has established that the level of scientific evidence of single-subject experimental designs (when some conditions are respected) has a rank that is similar to that of systematic reviews and just below that of a meta-analysis. Additionally the single-subject design is considered to have a higher ranking than the more conventional intergroup design often used for randomized clinical trials [51]. In the present study we integrate an experimental single-subject design that is repeated in multiple baselines. For the study an ample sample is available, and a significant number of data points is taken into account. This is made possible by the use of information technology, with activity trackers/wearables, which allow individual, continuous, and constant data collection.

This data analysis strategy is being performed with the intention-to-treat (ITT) method. This specific choice was adopted with two main purposes: maintaining similar treatment groups apart from randomization, and allowing non-compliance and deviations from the clinicians and researcher's policy, keeping most of the possible exceptions under control. The continuous sampling and data collection, made possible mainly by a technological system of automatic devices, makes the use of a large sample of subjects possible. Moreover, it allows an experimental analysis of the participants even with the methodology of the comparison between randomized groups. For the side of group analysis this study is a small size RCT, with four different experimental conditions, depending on two different independent variables, each on two levels (present or

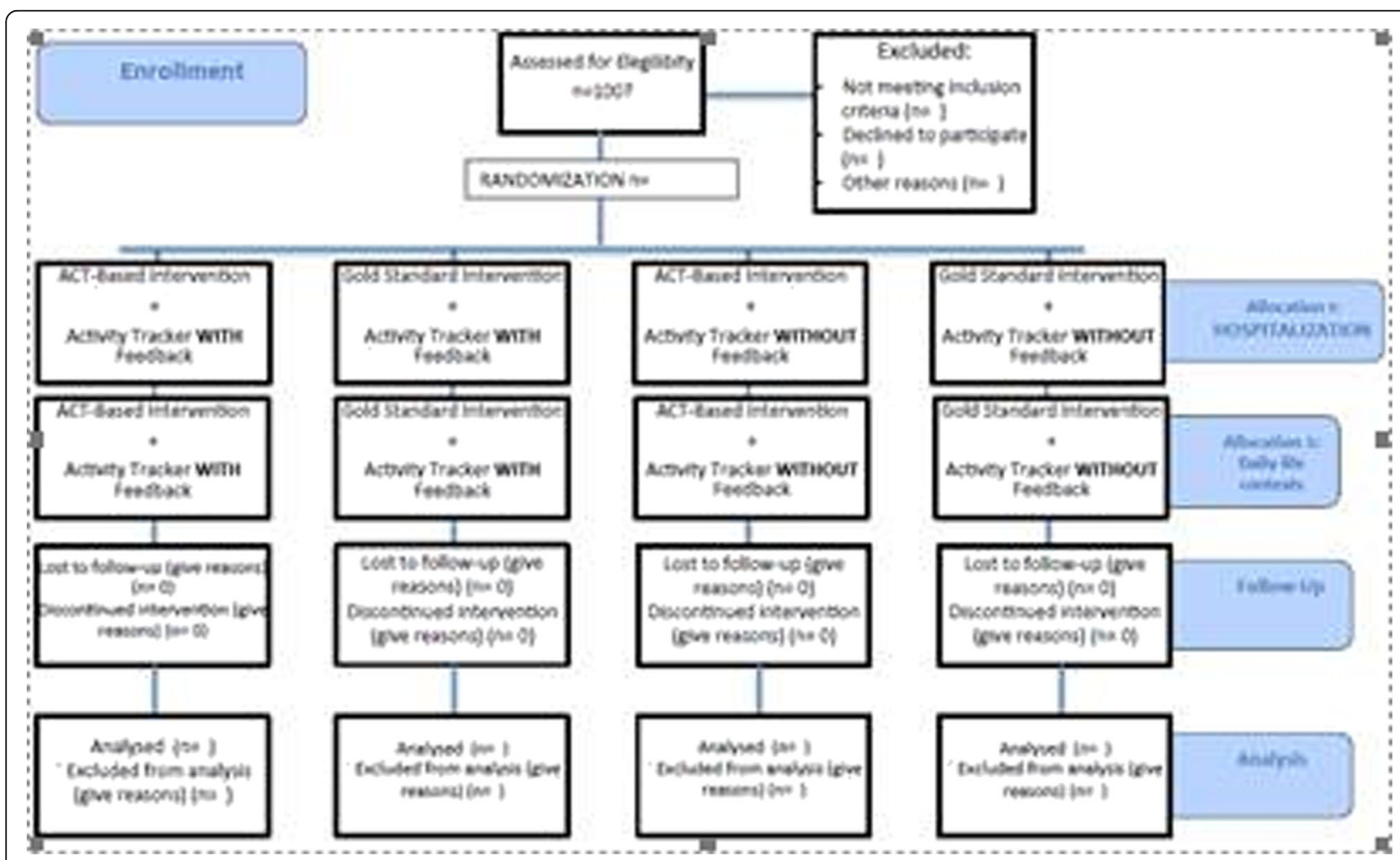

Fig. 1 CONSORT flow diagram 


\begin{tabular}{|c|c|c|c|c|c|c|c|}
\hline \multirow[b]{3}{*}{ TNePONT } & \multirow{3}{*}{$\begin{array}{l}\text { Etroimant } \\
\text { Doser }\end{array}$} & \multicolumn{6}{|c|}{ ITUOY PERDO } \\
\hline & & \multirow{2}{*}{ Aloseton } & \multicolumn{5}{|c|}{ 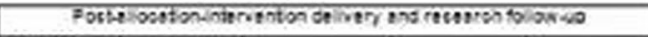 } \\
\hline & & & $\begin{array}{c}\text { dacsk } \\
\text { nuaveritor }\end{array}$ & 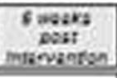 & $\begin{array}{l}3 \text { mosa: } \\
\text { nswritsen }\end{array}$ & $\begin{array}{l}6 \text { encost } \\
\text { manvesen }\end{array}$ & $12 m e s a r$ \\
\hline \multicolumn{8}{|l|}{ ENAOUVENT: } \\
\hline \multirow{3}{*}{ 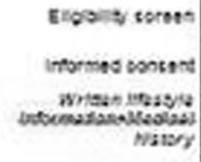 } & $x$ & & & & & & \\
\hline & $x$ & & & & & & \\
\hline & $x$ & & & & & & \\
\hline anlosestor & & $x$ & & & & & \\
\hline \\
\hline \multicolumn{8}{|l|}{ 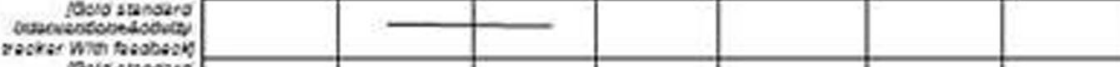 } \\
\hline 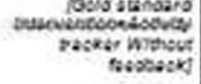 & & & & & & & \\
\hline 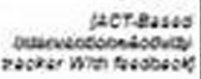 & & & & & & & \\
\hline
\end{tabular}

Fig. 2 SPIRIT figure

absent), with a classic $2 \times 2$ design factorial. For every condition all the participants of the study follow the same inpatient, 4-week rehabilitation program followed by a 16-week outpatient phase. Psychometric and direct measures are collected during both the inpatient and outpatient phases, and at follow-up at 3, 6, and 12 months.

The experimental conditions are as follows:

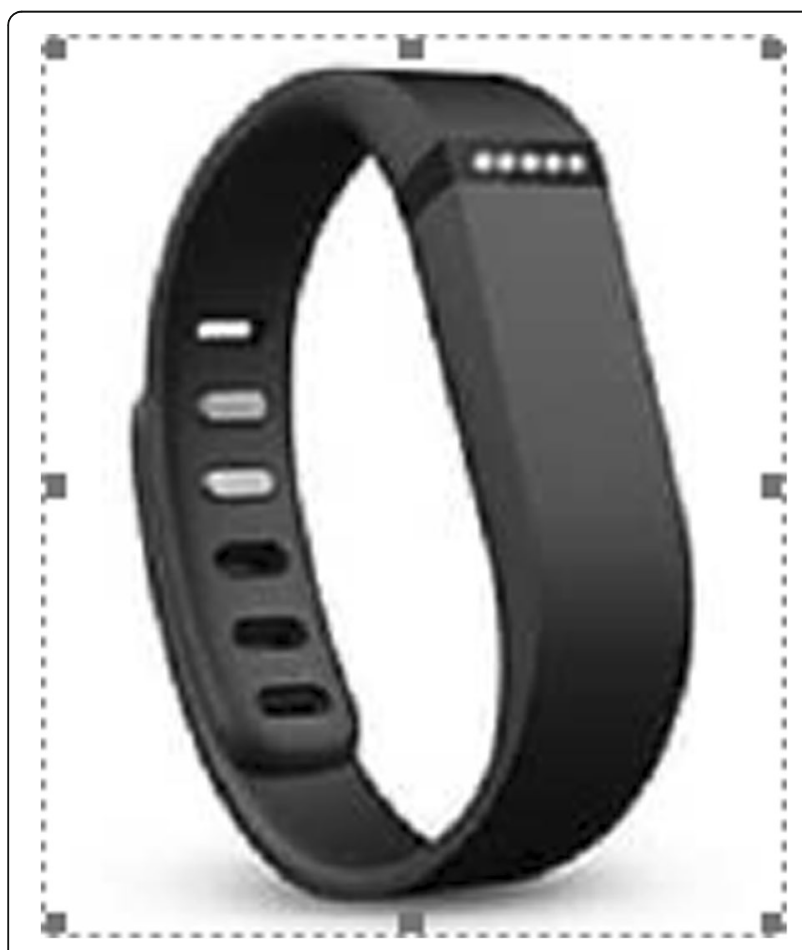

Fig. 3 Wearable sample
- Gold Standard Intervention plus Activity Tracker WITHOUT Feedback

- Gold Standard Intervention plus Activity Tracker WITH Feedback

- ACT-Based Intervention plus Activity Tracker WITHOUT Feedback

- ACT-Based Intervention plus Activity Tracker WITH Feedback

\section{Gold Standard Intervention plus Activity Tracker WITHOUT Feedback} Medical rehabilitation, motivational support, and psychoeducation

During the inpatient phase, participants will participate in the intensive 4-week hospital-based and medically managed rehabilitation program for weight reduction. All patients will be placed on a hypocaloric nutritionally balanced diet tailored to the individual after consultation with a dietitian. Furthermore, they will receive nutritional counseling provided by dietitians, physical activity training provided by physiotherapists, and motivational support with elements of psycho-education provided by physicians trained and informed by psychologistspsychotherapists.

The nutritional rehabilitation program aims to promote change in eating habits and consists of both individual and group sessions twice a week and includes information on obesity and related health risks, setting of realistic goals for weight loss, healthful eating in general, general nutrition and core food groups, weight management, and strategies for preventing relapse. The individual dietary counseling involves evaluation of nutrient intake and adequacy, nutritional and anthropometric status, dietary assessment, eating patterns, history of being overweight, and 
Table 1 Experimental conditions

\begin{tabular}{lll}
\hline & Brief ACT intervention & $\begin{array}{l}\text { Medical rehabilitation, motivational support, and } \\
\text { psycho-education }\end{array}$ \\
\hline $\begin{array}{lll}\text { Contingent and informative feedback (SR+) } \\
\text { provided by activity tracker }\end{array}$ & $\begin{array}{l}\text { ACT }+ \\
+ \text { Feedback provided by activity tracker }\end{array}$ & $\begin{array}{l}\text { No ACT - traditional motivational intervention } \\
+ \text { feedback provided by activity tracker }\end{array}$ \\
$\begin{array}{l}\text { Non-contingent and episodic feedback (SR+) } \\
-\begin{array}{l}\text { No feedback provided by activity tracker, } \\
\text { usual post-rehabilitation information }\end{array}\end{array}$ & ACT without feedback provided by activity tracker & $\begin{array}{l}\text { Traditional medical rehabilitation, motivational } \\
\text { support, and psycho-education without feedback } \\
\text { provided by activity tracker }\end{array}$ \\
\hline
\end{tabular}

SR+ states for Positive Reinforcement

readiness to adopt change. Another meeting will be scheduled to deliver the activity tracker and linked app. The trackers are presented as mere data collection devices, for medical use only, that do not provide any type of feedback or data to the participants.

\section{Gold Standard Intervention plus Activity Tracker WITH Feedback}

\section{Behavioral change condition with activity tracker}

In this experimental condition, participants will be provided the same rehabilitation program for the 4-week inpatient phase as in the control condition. In addition, for these subjects we will implement a stepped protocol using wearable devices/activity trackers to collect information about daily physical activity and providing meaningful and informative feedback. The additional procedure starts during the inpatient phase with delivery and explanation of the use of the wearable devices. In this meeting, longer than the one described for the control condition, experimenters provide information, set individualized goals, and explain the feedback to be delivered by the electronic wearable devices after the end of the inpatient phase. All subjects allocated to this condition will be clustered by date of entrance in the program; then, for each cluster, the length of the baseline phase will be randomly assigned. According to these allocations, the wearable device will start to provide feedback at different times for each participant in the cluster, to directly check the effect of contingent and informative feedback, one of the two independent variables of the study, on the daily level of physical activity, our primary outcome and dependent variable. The stepped protocol includes automatically generated feedback for achieving individualized goals in physical activity or related healthful habits. If the goal is missed one day, a daily report is automatically sent, but if any participant misses repeatedly, the individualized goal researchers provide an individualized contact by text message or e-mail.

\section{ACT-Based Intervention plus Activity Tracker WITHOUT Feedback}

ACT-based intervention

In this experimental condition, the subjects followe the normal medical rehabilitation program described above for the first experimental condition. In addition, within the 4-week period of the inpatient phase, we provide a brief ACT intervention during the hospitalization, followed by consulting/therapy sessions provided by a combination of phone calls, e-mails, and text messages in the outpatient phase. The total duration of one-to-one interventions during the hospitalization is $3 \mathrm{~h}$, with four 45 -min sessions during the rehabilitation. For the outpatient phase the ACT intervention includes a monthly 30 min Skype-telephone session, starting after the end of hospitalization, that includes weekly text messages or e-mails but is not linked with any kind of data regarding participants of the study.

The ACT-based interventions include the following: (1) acceptance or "willingness," which involves the active awareness of difficult private experiences without unnecessary and often dysfunctional attempts to control or avoid unpleasant emotions. "Acceptance" is the alternative to emotional and experiential avoidance. Participants will be trained to improve adherence to a healthful lifestyle by increasing willingness to experience unwanted and difficult sensations and urges while engaging in health-related behaviors. (2) Mindfulness is definable as the process by which we can engage in the present moment, adopting an open and curious attitude. Additionally, mindfulness aims to increase self-awareness by noticing all internal experiences, with a non-judging stance, becoming aware of judgments and being able to let them go. Mindfulness allows one to explore previous control-based attempts to cope with unpleasant thoughts, emotions, and sensations and to demonstrate the ineffectiveness of control while pursuing a meaningful life. (3) With the "defusion" process, participants will be encouraged to alleviate themselves from thoughts and feelings by turning attention toward the "noticing self," rather than sticking to difficult thoughts. (4) Identification of participant values and related behavioral goals will increase engagement in values-driven behavior, or committed action. Potential barriers to adopting values-driven behaviors and maintaining committed action in the long term will be explored and addressed through metaphors and experiential techniques.

\section{ACT-Based Intervention plus Activity Tracker WITH Feedback}

\section{Combining ACT and behavioral change}

In the last experimental condition, obese individuals will follow the same rehabilitation program in the inpatient 
phase of the behavioral change condition, with the addition of four brief ACT sessions of duration 45-min for a total amount of $3 \mathrm{~h}$ of one-to-one therapy sessions, exactly as in the ACT condition. In the 16-week outpatient phase, each participant receives feedback from an activity tracker following the same stepped protocol, but messages and feedback are informed by an ACT therapist, including values-based goal setting, the defusion process for difficult thoughts, mindfulness cues, and a set of ACT-consistent metaphors and messages.

Combining ACT, which is a technology itself [50], with an app and wearable technologies is quite innovative. Some commercial apps are already available, but they are not developed in the Italian language.

\section{Recruitment of the study population, inclusion and exclusion criteria}

Inpatients will be eligible when they will meet the following inclusion criteria at the admission to the hospital: (1) age between 18 and 70 years, (2) obesity according to the World Health Organization (WHO) criteria (body mass index (BMI) $\geq 30$ ), (3) provision of written and informed consent to participate, (4) technology friendly to receive feedback through smartphone, e-mail, and wearable devices. Exclusion criteria for the study are the following: (1) other severe psychiatric disturbance diagnosed by the Diagnostic and Statistical Manual of Mental Disorders (DSM-5) that causes functional impairment or might compromise treatment adherence criteria, (2) severe visual difficulties, (3) important limitations of movement in particular subjects for which physical activity is not recommended, (4) concurrent medical condition not related to obesity. DSM-5 [87] will be used as a screening tool for psychiatric disorders. The Medical Ethics Committee of Istituto Auxologico Italiano approved the study protocol and the informed consent process.

\section{Randomization procedure}

Using a double-blind procedure, we will randomly assign all of the participants to one of the four groups. The randomization scheme will be generated using the website Randomization.com (http://www.randomization.com). Randomization and follow-up assessments will be administered by independent practitioners blind to the research hypotheses and condition assignments. The G*Power software (v3.1.9.2) [35, 36] was used to compute the minimum sample size required to conduct this study. All of the participants will be assessed with the Psychological General Well-Being Index (PGWBI) [47, 62, 85] five times: (1) at admission to the clinic, (2) at discharge, (3) at 3 months follow-up, (4) at 6 months follow-up, and (5) at 12 months follow-up. Thus, the experimental condition is considered as a between-group variable, and time is considered as a within-subject variable. The a priori statistics (partial $\eta^{2}$ and correlation between measures) were computed by examining previous longitudinal studies using the PGWBI in subjects with obesity $[9,65]$. The a priori partial $\eta^{2}$ was set to assume a value of 0.015 (small effect size) [26, 30], the type I error $(\alpha)$ rate was set at 0.05 (two-sided), the power $(1-\beta)$ was set at 0.95 [26], and the a priori correlation between repeated measures was set at 0.50 . Finally, sphericity was assumed. G*Power showed that there is a $95 \%$ chance to correctly reject the null hypothesis of no significant effect with an overall sample of 168 participants, 42 subjects for each group.

\section{Assessment procedure}

According to the behavioral framework, direct measures are preferred to psychometric inventory when possible. In any case, to deepen the understanding of mechanisms of change, psychometric measures were included. Participants will be asked to complete all study measures before randomization. Follow-up repeated measures assessment will be completed on all study participants 6 weeks later (post-treatment) and at 3, 6, and 12 months follow-up.

Demographic data such as gender, age, marital status, level of education, and employment status will be assessed in order to control for these potential confounders, checking also for biological indicators of risk-obesity, according to WHO criteria, with a BMI of 30 or more [67].

Primary outcome is physical activity as assessed continuously by wearable technology. Activity trackers will collect data, by direct measurement, of daily steps, physical activity, sleep, and food journal with the self-report app connected with the wearable device. Medical data and additional diagnoses (e.g., diabetes) that may confound results will be retrieved from clinical records.

Participants in both groups will provide a history of any psychological treatment or counseling to assess potential familiarity with mindfulness and acceptance-based therapies. A range of physiological and psychological outcome measures will assess the following: modifiable risk factors and recommended lifestyle dietary habits with the Mediterranean Diet Score [73], physical exercise with the International Physical Activity Questionnaire - Short Form (IPAQ-SF) [57] and the Acceptance and Action Questionnaire for Exercise (AAQ-EX) [85]. We also collect data about psychological well-being and quality of life (PGWBI) [47, 62, 85] and psychological flexibility (AAQ-II) $[4,49]$, a core process directly targeted by ACT interventions and involved as a mediator for well-being [53]. Adjusted change scores for all the outcome measures will be calculated in order to assess the ability of the interventions to produce changes over time.

\section{Psychotherapists and treatment fidelity}

Motivational support and psycho-education will be supervised by experienced and certified psychotherapists 
from diverse backgrounds with specific training in CBT. The brief ACT intervention will be delivered by two psychotherapists with ACT and CBT background and formal training in ACT. They will receive monthly supervision by senior certified psychotherapists to ensure competent and uniform treatment delivery. A fidelity checklist, checked by a third, blinded therapist, will also be used for both treatments.

\section{Statistical analysis}

As this study combines group design with different single-case design or, more in detail, randomly assigned multiple baseline design clustered by sub-sequential recruitment. Following methodological guidelines of RCT, the implementation of statistical analysis is linked to synergies that we can not previse at this point.

Statistical analyses will be performed using the R software [86]. Preliminary analyses will be carried out to assess the assumptions of parametric statistics; in case of violations, robust methods or data transformation will be used. Direct collected data (steps, weight, etc.) will be analyzed with an ITT approach with dropouts assumed to have regained $0.3 \mathrm{~kg}$ per month, an assumption already used in previous studies [16]. Dropouts will be excluded from the study.

The analysis of variance (ANOVA) will be used to examine between-group differences for all the primary and secondary outcomes at all time-points, focusing on the change in the scores. Corrected effect sizes (Hedges' $g$ ) and 95\% confidence interval (95\% CI) will be calculated for both between-group and withingroup differences.

Moreover, growth curve model analysis with a group variable (between conditions) will be used to evaluate the average growth from the baseline to 12 months after treatment for all of the outcome variables as performed in previous studies [52]. Moderation and meditational analysis will be implemented as more advanced statistical models. All data analyses will be performed using the Statistical Package for the Social Sciences (version 16.0; SPSS, Inc., Chicago, IL).

In addition, considering that the present study combines different single-case designs, more detailed randomly assigned multiple baseline designs clustered by sub-sequential recruitment, and a group design following methodological guidelines of RCTs, the implementation of statistical analysis is linked to synergies that we cannot previse at this point. For the single-case design, we use a multiple-baseline procedure with the same number of data points for all the subjects assigned to a cluster. In each multiple-baseline cluster we randomly assign the length of the baseline and, consequently, of the treatment. Detailed visual inspection and graphical analysis will be use, followed by a randomization test to ensure statistical power to the visual analysis $[37,38]$.

\section{Discussion}

Due to the novelty of using a combination of behavioral change strategies with acceptance and mindfulness-based therapy with a wearable device to promote a healthful lifestyle in the post-rehabilitation period, no well-defined hypotheses have been established about the possible contribution of this kind of intervention in comparison with the "gold standard" multidisciplinary treatment chronic conditions linked to obesity. With the development of mobile technology and brief clinical protocols, new interventions in the field of obesity and connected chronic pathologies have to be evaluated.

The mACTonHEALTH study is quite innovative for different reasons. First, the combination of single-case designs with an RCT is uncommon, particularly with medium-scale studies. Second, the use of a wearable device activity tracker both as a device for data collection measuring the primary outcomes and as a device to provide contingent feedback, assumed as one of the two independent variables, is unique. Third, directly combining brief ACT intervention and behavior modification strategies with mobile-tech implementation is another element of innovation. Furthermore, although ACT is becoming well studied in the traditional clinical context, large-scale application to both medical and natural settings has not largely occurred. Including in the assessment procedure directly collectable data as indicators of both physical and psychosocial health is unique. Also, the inclusion of objective physiological outcome measures constitutes an aspect of innovation in ACT research. Last, the study will focus on mediation mechanisms underlying the treatment's success and present possible links to systematic models on psychological flexibility, a core process assessed in ACT research.

The particular experimental design, using both directly measured outcomes and psychometric measures, particularly aimed at checking for mediation and moderation effects, is also one of the limits of the study. The complex experimental design with multiple independent variables requires a higher number of participants and could weaken the findings if the conclusions are not clear. Another limit of the study is represented by the absence of a real group without activity trackers. This decision is due to the importance of gathering data only collectable by the trackers, but even when the wearables collect data in "silent" mode, without providing any feedback, participants wear the devices. Even assuming that the effect of simply wearing a similar device is small, we cannot check directly. Despite these limitations and others of lesser importance, such as the limited number of psychometric inventories and the relatively small 
sample size of each subgroup, the benefits of this experimental design seem to overcome the downsides. First of all, direct measure of movement and physical activity represents an essential outcome, rarely collected in such a sample. Moreover, despite the downside of a small sample, a similar method could be used as an efficient way to test different hypotheses and to control for interactions between variables.

To the best of the researcher's knowledge, this study will be the first RCT to examine the effectiveness of combining contingent feedback provided with wearable devices with ACT-based interventions to make the lifestyle more healthful according to medical and behavioral prescription among obese patients. This study is also one of the first examples of a large implementation of multiple single-case designs with a continuous data collection, made possible through the implementation of wearable technology. Conducted in the context of clinical practice, this trial will potentially offer empirical support to alternative interventions to improve radical long-time change in life habits and consequently promote quality of life, reducing mortality and morbidity rates among an obese population. Furthermore, by virtue of its short duration, the program could potentially also be utilized for health promotion in non-hospitalization contexts and primary or secondary prevention of obesity and linked conditions.

\section{Additional files}

Additional file 1: The TIDieR (Template for Intervention Description and Replication) checklist*. (PDF $273 \mathrm{~kb}$ )

Additional file 2: CONSORT 2010 checklist of information to include when reporting a randomised trial*. (DOC $218 \mathrm{~kb}$ )

Additional file 3: SPIRIT 2013 checklist: recommended items to address in clinical trial protocol and related documents*. (PNG $601 \mathrm{~kb}$ )

\section{Acknowledgements}

The contribution of Emanuela Bezzoli for the selection of the outcome measures is gratefully acknowledged.

\section{Funding}

The trial will be self-funded by the investigators.

\section{Availability of data and materials}

The datasets generated and/or analyzed during the current study will be available from the corresponding author on request. Records of all patients will be kept separately in a secure place.

\section{Authors' contributions}

$\mathrm{RC}$ conceived the study, planned the experimental design and made substantial contribution to the manuscript drafting. GC, AM, GV and CAMS participated in the development of the study design and contributed greatly to the manuscript drafting. FVR, FT, AGU, GMM, PC, GP and EM participated in the study design and helped to draft the manuscript. AR defined the statistical analysis we are planning to use for the data collected and establish the sample size for the study. All authors read and approved the final manuscript.

\section{Ethics approval and consent to participate}

Ethical approval for this trial was obtained from the Ethics Committee of "Istituto Auxologico Italiano" (V.0.4 30-05-2017). The study personnel and the Ethics Committee will ensure that the study is conducted within appropriate professional ethical guidelines and that Good Clinical Practice guidelines are observed. All participants provide written informed consent before participating in the trial or extension study.

\section{Consent for publication}

Not applicable.

\section{Competing interests}

The authors declare that they have no competing interests.

\section{Publisher's Note}

Springer Nature remains neutral with regard to jurisdictional claims in published maps and institutional affiliations.

\section{Author details}

'Istituto Auxologico Italiano IRCCS, Psychology Research Laboratory, Verbania, Italy. ${ }^{2}$ Department of Psychology, Catholic University of Milan, Milan, Italy. ${ }^{3}$ Department of Humanities, Social Sciences and Cultural Industries, University of Parma, Parma, Italy. ${ }^{4}$ Faculty of Psychology, eCampus University, Novedrate, Italy. ${ }^{5}$ AARBA, Association for the Advancement of Radical Behavior Analysis, Milan, Italy. ${ }^{6}$ Interdepartmental Center for Family Research, Department of Philosophy, Sociology, Education, and Applied Psychology, University of Padova, Padova, Italy. ${ }^{7}$ Istituto Auxologico Italiano IRCCS, Rehabilitation Unit and Research Laboratory of Biomechanics and Rehabilitation, S Giuseppe, Piancavallo, Oggebbio, Verbania, Italy.

Received: 15 December 2017 Accepted: 8 October 2018

Published online: 29 November 2018

\section{References}

1. Adam Noah J, Spierer DK, Gu J, Bronner S. Comparison of steps and energy expenditure assessment in adults of Fitbit Tracker and Ultra to the Actical and indirect calorimetry. J Med Eng Technol. 2013;37(7):456-62. https://doi. org/10.3109/03091902.2013.831135

2. Aldridge D. Single-case research designs for the clinician. J R Soc Med. 1991; 84(5):249-52.

3. A-Tjak JG, Davis ML, Morina N, Powers MB, Smits JA, Emmelkamp PM. A meta-analysis of the efficacy of acceptance and commitment therapy for clinically relevant mental and physical health problems. Psychother Psychosom. 2015:84(1):30-6. https://doi.org/10.1159/000365764.

4. Avena NM, Bocarsly ME, Hoebel BG, Gold MS. Overlaps in the nosology of substance abuse and overeating: the translational implications of "food addiction". Curr Drug Abuse Rev. 2011;4(3):133-9.

5. Avena NM, Gold JA, Kroll C, Gold MS. Further developments in the neurobiology of food and addiction: update on the state of the science. Nutrition. 2012;28(4):341-3. https://doi.org/10.1016/j.nut.2011.11.002.

6. Baranoff JA, Hanrahan SJ, Burke AL, Connor JP. Changes in acceptance in a low-intensity, group-based acceptance and commitment therapy (ACT) chronic pain intervention. Int J Behav Med. 2015. https://doi.org/10.1007/ s12529-015-9496-9.

7. Barnes RD, Tantleff-Dunn S. Food for thought: examining the relationship between food thought suppression and weight-related outcomes. Eat Behav. 2010;11(3):175-9. https://doi.org/10.1016/j.eatbeh.2010.03.001.

8. Behi R, Nolan M. Single-case experimental designs. 1: Using idiographic research. Br J Nurs. 1996:5(21):1334-7. https://doi.org/10.12968/bjon.1996.5.21.1334.

9. Bertella L, Mori I, Grugni G, Pignatti R, Ceriani F, Molinari E, et al. Quality of life and psychological well-being in GH-treated, adult PWS patients: a longitudinal study. J Intellect Disabil Res. 2007;51(4):302-11. https://doi.org/ 10.1111/j.1365-2788.2006.00878.x

10. Bricker J, Wyszynski C, Comstock B, Heffner JL. Pilot randomized controlled trial of web-based acceptance and commitment therapy for smoking cessation. Nicotine Tob Res. 2013;15(10):1756-64. https:/doi.org/10.1093/ntr/ntt056.

11. Byrne S, Cooper Z, Fairburn C. Weight maintenance and relapse in obesity: a qualitative study. Int J Obes Relat Metab Disord. 2003;27(8):955-62. https://doi.org/10.1038/sj.ijo.0802305.

12. Cadmus-Bertram L, Marcus BH, Patterson RE, Parker BA, Morey BL. Use of the Fitbit to measure adherence to a physical activity intervention among 
overweight or obese, postmenopausal women: self-monitoring trajectory during 16 weeks. JMIR Mhealth Uhealth. 2015;3(4):e96. https://doi.org/10. 2196/mhealth.4229.

13. Callaghan GM, Duenas JA, Nadeau SE, Darrow SM, Van der Merwe J, Misko J. An empirical model of body image disturbance using behavioral principles found in functional analytic psychotherapy and acceptance and commitment therapy. Int J Behav Consult Ther. 2012;7(2-3):16-24.

14. Carr KA, Daniel TO, Lin H, Epstein LH. Reinforcement pathology and obesity. Curr Drug Abuse Rev. 2011;4(3):190-6.

15. Castelnuovo G, Manzoni GM, Pietrabissa G, Corti S, Giusti EM, Molinari E, Simpson S. Obesity and outpatient rehabilitation using mobile technologies: the potential mHealth approach. Front Psychol. 2014;5:559. https://doi.org/ 10.3389/fpsyg.2014.00559.

16. Castelnuovo G, Manzoni GM, Villa V, Cesa GL, Pietrabissa G, Molinari E. The STRATOB study: design of a randomized controlled clinical trial of Cognitive Behavioral Therapy and Brief Strategic Therapy with telecare in patients with obesity and binge-eating disorder referred to residential nutritional rehabilitation. Trials. 2011;12:114. https://doi.org/10.1186/1745-6215-12-114.

17. Castelnuovo G, Pietrabissa G, Cattivelli R, Manzoni GM, Molinari E. Not only clinical efficacy in psychological treatments: clinical psychology must promote cost-benefit, cost-effectiveness, and cost-utility analysis. Front Psychol. 2016;7:563. https://doi.org/10.3389/fpsyg.2016.00563.

18. Castelnuovo G, Pietrabissa G, Manzoni GM, Cattivelli R, Rossi A, Novelli M, et al. Cognitive behavioral therapy to aid weight loss in obese patients: current perspectives. Psychol Res Behav Manag. 2017;10:165-73. https://doi. org/10.2147/PRBM.S113278.

19. Castelnuovo G, Simpson S. Ebesity - e-health for obesity - new technologies for the treatment of obesity in clinical psychology and medicine. Clin Pract Epidemiol Ment Health. 2011;7:5-8. https://doi.org/10. 2174/1745017901107010005.

20. Castelnuovo G, Zoppis I, Santoro E, Ceccarini M, Pietrabissa G, Manzoni GM, et al. Managing chronic pathologies with a stepped mHealth-based approach in clinical psychology and medicine. Front Psychol. 2015;6:407. https://doi.org/10.3389/fpsyg.2015.00407.

21. Cattivelli R, Cavallini F, Tirelli V. Prospettive educative attraverso un approccio clinico: i contributi dell'Acceptance and Commitment Therapy e della Functional Analytic Psychotherapy nel caso di un ragazzo con ansia sociale. Psicoterapia Cognitivo Comportamentale. 2012;18(3).

22. Cattivelli R, Pietrabissa G, Ceccarini M, Spatola CA, Villa V, Caretti A, et al. ACTonFOOD: opportunities of ACT to address food addiction. Front Psychol. 2015;6:396. https://doi.org/10.3389/fpsyg.2015.00396.

23. Cattivelli R, Tirelli V, Berardo F, Perini S. Promoting appropriate behavior in daily life contexts using functional analytic psychotherapy in earlyadolescent children. Int J Behav Consult Therapy. 2012;7:25-32.

24. Cawley J, Meyerhoefer C, Biener A, Hammer M, Wintfeld N. Savings in medical expenditures associated with reductions in body mass index among US adults with obesity, by diabetes status. PharmacoEconomics. 2014. https://doi.org/10.1007/s40273-014-0230-2.

25. Cesa GL, Manzoni GM, Bacchetta M, Castelnuovo G, Conti S, Gaggioli A, et al. Virtual reality for enhancing the cognitive behavioral treatment of obesity with binge eating disorder: randomized controlled study with one-year follow-up. J Med Internet Res. 2013;15(6):e113. https://doi.org/10.2196/jmir.2441.

26. Cohen J. Statistical power analysis for the behavioral sciences. 2nd ed. Hillsdale: Lawrence Erlbaum Associates; 1988.

27. Cooper Z, Doll HA, Hawker DM, Byrne S, Bonner G, Eeley E, et al. (2010). Testing a new cognitive behavioural treatment for obesity: a randomized controlled trial with three-year follow-up. Behav Res Ther, 48(8), 706-713. doi:https://doi.org/10.1016/j.brat.2010.03.008.

28. Curioni CC, Lourenço PM. Long-term weight loss after diet and exercise: a systematic review. Int J Obes. 2005;29(10):1168-74. https://doi.org/10.1038/ sj.ijo.0803015.

29. Deitel M. The International Obesity Task Force and "globesity". Obes Surg. 2002;12(5):613-4.

30. Eid M, Gollwitzer M, Schmitt M. Statistik und Forschungsmethoden. 5th ed Basel: Beltz; 2017.

31. Epstein LH, Lin $H$, Carr KA, Fletcher KD. Food reinforcement and obesity. Psychological moderators. Appetite. 2012;58(1):157-62. https://doi.org/10. 1016/j.appet.2011.09.025

32. Epstein LH, Salvy SJ, Carr KA, Dearing KK, Bickel WK. Food reinforcement, delay discounting and obesity. Physiol Behav. 2010;100(5):438-45. https:// doi.org/10.1016/j.physbeh.2010.04.029.
33. Evenson KR, Goto MM, Furberg RD. Systematic review of the validity and reliability of consumer-wearable activity trackers. Int J Behav Nutr Phys Act. 2015;12:159. https://doi.org/10.1186/s12966-015-0314-1.

34. Fanning J, Walkup MP, Ambrosius WT, Brawley LR, Ip EH, Marsh AP, Rejeski WJ. Change in health-related quality of life and social cognitive outcomes in obese, older adults in a randomized controlled weight loss trial: does physical activity behavior matter? J Behav Med. 2017. https://doi.org/10. 1007/s10865-017-9903-6.

35. Faul F, Erdfelder E, Buchner A, Lang AG. Statistical power analyses using G*Power 3.1: tests for correlation and regression analyses. Behav Res Methods. 2009;41(4):1149-60. https://doi.org/10.3758/BRM.41.4.1149.

36. Faul F, Erdfelder E, Lang AG, Buchner A. G*Power 3: a flexible statistical power analysis program for the social, behavioral, and biomedical sciences. Behav Res Methods. 2007;39(2):175-91.

37. Ferron J, Ware W. Using randomization tests with responsive single-case designs. Behav Res Ther. 1994;32(7):787-91.

38. Fisher WW, Kelley ME, Lomas JE. Visual aids and structured criteria for improving visual inspection and interpretation of single-case designs. J Appl Behav Anal. 2003;36(3):387-406. https://doi.org/10.1901/jaba.2003.36-387.

39. Foreyt JP, Poston WS. Obesity: a never-ending cycle? Int J Fertil Womens Med. 1998a;43(2):111-6.

40. Foreyt JP, Poston WS. What is the role of cognitive-behavior therapy in patient management? Obes Res. 1998b;6(Suppl 1):18S-22S.

41. Forman EM, Hoffman KL, Juarascio AS, Butryn ML, Herbert JD. Comparison of acceptance-based and standard cognitive-based coping strategies for craving sweets in overweight and obese women. Eat Behav. 2013;14(1):648. https://doi.org/10.1016/j.eatbeh.2012.10.016.

42. Forman EM, Hoffman KL, McGrath KB, Herbert JD, Brandsma LL, Lowe MR. A comparison of acceptance- and control-based strategies for coping with food cravings: an analog study. Behav Res Ther. 2007:45(10):2372-86. https://doi.org/10.1016/j.brat.2007.04.004.

43. Fossati M, Amati F, Painot D, Reiner M, Haenni C, Golay A. Cognitivebehavioral therapy with simultaneous nutritional and physical activity education in obese patients with binge eating disorder. Eat Weight Disord. 2004;9(2):134-8.

44. Gallus S, Lugo A, Murisic B, Bosetti C, Boffetta P, La Vecchia C. Overweight and obesity in 16 European countries. Eur J Nutr. 2015;54(5):679-89. https:// doi.org/10.1007/s00394-014-0746-4.

45. García-García I, Horstmann A, Jurado MA, Garolera M, Chaudhry SJ, Margulies DS, et al. Reward processing in obesity, substance addiction and non-substance addiction. Obes Rev. 2014;15(11):853-69. https://doi.org/10. 1111/obr.12221.

46. Gifford EV, Lillis J. Avoidance and inflexibility as a common clinical pathway in obesity and smoking treatment. J Health Psychol. 2009;14(7):992-6. https://doi.org/10.1177/1359105309342304.

47. Grossi E, Groth N, Mosconi P, Cerutti R, Pace F, Compare A, Apolone G. Development and validation of the short version of the Psychological General Well-Being Index (PGWB-S). Health Qual Life Outcomes. 2006;4:88. https://doi.org/10.1186/1477-7525-4-88.

48. Hayes S, Hogan M, Dowd H, Doherty E, O'Higgins S, Nic Gabhainn S, MacNeela P, Murphy AW, Kropmans T, O'Neill C, Newell J, McGuire BE. Comparing the clinical-effectiveness and cost-effectiveness of an internetdelivered Acceptance and Commitment Therapy (ACT) intervention with a waiting list control among adults with chronic pain: study protocol for a randomised controlled trial. BMJ Open. 2014;4(7):e005092. https://doi.org/10. 1136/bmjopen-2014-005092.

49. Hayes SC, Luoma JB, Bond FW, Masuda A, Lillis J. Acceptance and commitment therapy: model, processes and outcomes. Behav Res Ther. 2006:44(1):1-25. https://doi.org/10.1016/j.brat.2005.06.006.

50. Hayes SC, Wilson KG. Acceptance and commitment therapy: altering the verbal support for experiential avoidance. Behav Anal. 1994;17(2):289-303.

51. Howick J, Glasziou P, Aronson JK. The evolution of evidence hierarchies: what can Bradford Hill's 'guidelines for causation' contribute? J R Soc Med. 2009;102(5):186-94. https://doi.org/10.1258/jrsm.2009.090020.

52. Jackson JB, Pietrabissa G, Rossi A, Manzoni GM, Castelnuovo G. Brief strategic therapy and cognitive behavioral therapy for women with binge eating disorder and comorbid obesity: a randomized clinical trial one-year follow-up. J Consult Clin Psychol. 2018;86(8):688-701. https://doi.org/10. 1037/ccp0000313.

53. Kashdan TB, Barrios V, Forsyth JP, Steger MF. Experiential avoidance as a generalized psychological vulnerability: comparisons with coping and 
emotion regulation strategies. Behav Res Ther. 2006;44(9):1301-20. https:// doi.org/10.1016/j.brat.2005.10.003.

54. Kashikar-Zuck S, Flowers SR, Strotman D, Sil S, Ting TV, Schikler KN. Physical activity monitoring in adolescents with juvenile fibromyalgia: findings from a clinical trial of cognitive-behavioral therapy. Arthritis Care Res (Hoboken). 2013;65(3):398-405. https://doi.org/10.1002/acr.21849.

55. Knäuper B, Ivanova E, Xu Z, Chamandy M, Lowensteyn I, Joseph L, Luszczynska A, Grover S. Increasing the effectiveness of the Diabetes Prevention Program through if-then plans: study protocol for the randomized controlled trial of the McGill CHIP Healthy Weight Program. BMC Public Health. 2014;14:470. https://doi.org/10.1186/1471-2458-14-470.

56. Kramer MK, McWilliams JR, Chen HY, Siminerio LM. A community-based diabetes prevention program: evaluation of the group lifestyle balance program delivered by diabetes educators. Diabetes Educ. 2011;37(5):659-68. https://doi.org/10.1177/0145721711411930.

57. Lee PH, Macfarlane DJ, Lam TH, Stewart SM. Validity of the international physical activity questionnaire short form (IPAQ-SF): a systematic review. Int J Behav Nutr Phys Activ. 2011;8(1):115.

58. Lehnert T, Streltchenia P, Konnopka A, Riedel-Heller SG, König HH. Health burden and costs of obesity and overweight in Germany: an update. Eur J Health Econ. 2014. https://doi.org/10.1007/s10198-014-0645-x.

59. Lifshitz F, Lifshitz JZ. Globesity: the root causes of the obesity epidemic in the USA and now worldwide. Pediatr Endocrinol Rev. 2014;12(1):17-34.

60. Lillis J, Hayes SC, Bunting K, Masuda A. Teaching acceptance and mindfulness to improve the lives of the obese: a preliminary test of a theoretical model. Ann Behav Med. 2009;37(1):58-69. https://doi.org/10. 1007/s12160-009-9083-x

61. Lillis J, Hayes SC, Levin ME. Binge eating and weight control: the role of experiential avoidance. Behav Modif. 2011;35(3):252-64. https://doi.org/10. $1177 / 0145445510397178$

62. Lundgren-Nilsson $\AA$, Jonsdottir $H$, Ahlborg G, Tennant A. Construct validity of the Psychological General Well Being Index (PGWBI) in a sample of patients undergoing treatment for stress-related exhaustion: a Rasch analysis. Health Qual Life Outcomes. 2013;11:2. https://doi.org/10.1186/14777525-11-2.

63. Manzoni GM, Pagnini F, Corti S, Molinari E, Castelnuovo G. Internet-based behavioral interventions for obesity: an updated systematic review. Clin Pract Epidemiol Ment Health. 2011;7:19-28. https://doi.org/10.2174/ 1745017901107010019

64. Manzoni GM, Pagnini F, Gorini A, Preziosa A, Castelnuovo G, Molinari E, Riva G. Can relaxation training reduce emotional eating in women with obesity? An exploratory study with 3 months of follow-up. J Am Diet Assoc. 2009; 109(8):1427-32. https://doi.org/10.1016/j.jada.2009.05.004.

65. Manzoni GM, Villa V, Compare A, Castelnuovo G, Nibbio F, Titon AM, Molinari E, Gondoni LA. Short-term effects of a multi-disciplinary cardiac rehabilitation programme on psychological well-being, exercise capacity and weight in a sample of obese in-patients with coronary heart disease: A practice-level study. Psychol Health Med. 2011;16(2):178-89. https://doi.org/ 10.1080/13548506.2010.542167.

66. McCracken LM, Jones R. Treatment for chronic pain for adults in the seventh and eighth decades of life: a preliminary study of Acceptance and Commitment Therapy (ACT). Pain Med. 2012;13(7):860-7. https://doi.org/10. 1111/j.1526-4637.2012.01407.x.

67. Meule A, Gearhardt AN. Food addiction in the light of DSM-5. Nutrients. 2014;6(9):3653-71. https://doi.org/10.3390/nu6093653.

68. Moulos I, Maramis C, Mourouzis A, Maglaveras N. Designing the user interfaces of a behavior modification intervention for obesity \& eating disorders prevention. Stud Health Technol Inform. 2015;210:647-51.

69. Musetti A, Cattivelli R, Giacobbi M, Zuglian P, Ceccarini M, Capelli F, Castelnuovo G. Challenges in internet addiction disorder: is a diagnosis feasible or not?. Front Psychol. 2016;7:842. https://doi.org/10.1421/87345.

70. Musetti A, Cattivelli R, Zuglian P, Terrone G, Pozzoli S, Capelli F, Castelnuovo G. Internet addiction disorder or internet related pPsychopathology?. Giorn It di Psic. 2017:44(2):359-82. https://doi.org/10.3389/fpsyg.2016.00842.

71. Nielsen D. Behavior-based safety in health care environments. G Ital Med Lav Ergon. 2010;32(1 Suppl A):A33-4.

72. Ostendorf DM, Lyden K, Pan Z, Wyatt HR, Hill JO, Melanson EL, Catenacci VA. Objectively measured physical activity and sedentary behavior in successful weight loss maintainers. Obesity (Silver Spring). 2017. https://doi. org/10.1002/oby.22052.
73. Panagiotakos DB, Pitsavos C, Stefanadis C. Dietary patterns: a Mediterranean diet score and its relation to clinical and biological markers of cardiovascular disease risk. Nutr Metab Cardiovasc Dis. 2006;16(8):559-68. https://doi.org/10.1016/j.numecd.2005.08.006.

74. Pietrabissa G, Manzoni GM, Corti S, Vegliante N, Molinari E, Castelnuovo G. Addressing motivation in globesity treatment: a new challenge for clinical psychology. Front Psychol. 2012;3:317. https://doi.org/10.3389/ fpsyg.2012.00317.

75. Pietrabissa G, Sorgente A, Rossi A, Simpson S, Riva G, Manzoni GM, et al. (2017). Stages of change in obesity and weight management: factorial structure of the Italian version of the University of Rhode Island Change Assessment Scale. Eat Weight Disord, 22(2), 361-367. doi:https://doi.org/10. 1007/s40519-016-0289-1.

76. Rachlin H. Teleological behaviorism. Am Psychol. 1992;47(11):1371-82.

77. Rachlin H. Self and self-control. Ann N Y Acad Sci. 1997;818:84-97.

78. Rachlin H. Free will from the viewpoint of teleological behaviorism. Behav Sci Law. 2007;25(2):235-50. https://doi.org/10.1002/bsl.746.

79. Rachlin H, Krasnoff J. Eating and drinking: an economic analysis. J Exp Anal Behav. 1983;39(3):385-404.

80. Riva G, Bacchetta M, Cesa G, Conti S, Castelnuovo G, Mantovani F, Molinari E. Is severe obesity a form of addiction? Rationale, clinical approach, and controlled clinical trial. Cyberpsychol Behav. 2006;9(4):457-79. https://doi. org/10.1089/cpb.2006.9.457.

81. Schuck K, Otten R, Kleinjan M, Bricker JB, Engels RC. Self-efficacy and acceptance of cravings to smoke underlie the effectiveness of quitline counseling for smoking cessation. Drug Alcohol Depend. 2014;142:269-76. https://doi.org/10.1016/j.drugalcdep.2014.06.033.

82. Sorgente A, Pietrabissa G, Manzoni GM, Re F, Simpson S, Perona S, Rossi A, Cattivelli R, Innamorati M, Jackson JB, Castelnuovo G. Web-based interventions for weight loss or weight loss maintenance in overweight and obese people: a systematic review of systematic reviews. J Med Internet Res. 2017;19(6):e229. https://doi.org/10.2196/jmir.6972.

83. Spatola CA, Manzoni GM, Castelnuovo G, Malfatto G, Facchini M, Goodwin $\mathrm{CL}$, Baruffi $\mathrm{M}$, Molinari E. The ACTonHEART study: rationale and design of a randomized controlled clinical trial comparing a brief intervention based on Acceptance and Commitment Therapy to usual secondary prevention care of coronary heart disease. Health Qual Life Outcomes. 2014;12:22. https:// doi.org/10.1186/1477-7525-12-22

84. Specchia ML, Veneziano MA, Cadeddu C, Ferriero AM, Mancuso A, lanuale C, Parente P, Capri S, Ricciardi W. (2014). Economic impact of adult obesity on health systems: a systematic review. Eur J Pub Health 25, 255-262. doi: https://doi.org/10.1093/eurpub/cku170.

85. Staats SB. Development and validation of the Acceptance and Action Questionnaire for Exercise. Minneapolis, Minnesota: Paper presented at the World Conference of the Association for Contextual Behavioral Science; 2014.

86. R Development Core Team. R: A Language and Environment for Statistical Computing. Vienna: R Foundation for Statistical Computing; 2014. http://www.R-project.org/

87. Thomas JJ, Koh KA, Eddy KT, Hartmann AS, Murray HB, Gorman MJ, Sogg S, Becker AE. Do DSM-5 eating disorder criteria overpathologize normative eating patterns among individuals with obesity? J Obes. 2014;2014:320803. https://doi.org/10.1155/2014/320803.

88. Tosolin F. Behavior-based safety. G Ital Med Lav Ergon. 2010;32(1 Suppl A):A7-12.

89. Tremmel M, Gerdtham UG, Nilsson PM, Saha S. Economic burden of obesity: a systematic literature review. Int J Environ Res Public Health. 2017;14(4). https://doi.org/10.3390/ijerph14040435.

90. Unick JL, Beavers D, Bond DS, Clark JM, Jakicic JM, Kitabchi AE, Knowler WC, Wadden TA, Wagenknecht LE, Wing RR, Look AHEAD Research Group. The long-term effectiveness of a lifestyle intervention in severely obese individuals. Am J Med. 2013;126(3):236-242, 242.e231-232. https://doi.org/ 10.1016/j.amjmed.2012.10.010.

91. Vancampfort D, Probst M, Adriaens A, Pieters G, De Hert M, Stubbs B, Soundy A, Vanderlinden J. Changes in physical activity, physical fitness, selfperception and quality of life following a 6-month physical activity counseling and cognitive behavioral therapy program in outpatients with binge eating disorder. Psychiatry Res. 2014;219(2):361-6. https://doi.org/10. 1016/j.psychres.2014.05.016. 